\title{
Resonance phenomena at the long wave run-up on the coast
}

\author{
A. Ezersky ${ }^{1}$, D. Tiguercha ${ }^{1}$, and E. Pelinovsky ${ }^{2,3,4,5}$ \\ ${ }^{1}$ CNRS UMR6143 Morphodynamique Continentale et Côtière (M2C), Université Caen Basse-Normandie, \\ 24 rue des Tilleuls, 14000 Caen, France \\ ${ }^{2}$ Institute of Applied Physics, Nizhny Novgorod, Russia \\ ${ }^{3}$ Nizhny Novgorod State Technical University, Nizhny Novgorod, Russia \\ ${ }^{4}$ National Research University - Higher School of Economics, Nizhny Novgorod, Russia \\ ${ }^{5}$ Johannes Kepler University, Linz, Austria \\ Correspondence to: E. Pelinovsky (pelinovsky@hydro.appl.sci-nnov.ru)
}

Received: 5 March 2013 - Published in Nat. Hazards Earth Syst. Sci. Discuss.: 21 March 2013

Revised: 13 September 2013 - Accepted: 29 September 2013 - Published: 5 November 2013

\begin{abstract}
Run-up of long waves on a beach consisting of three pieces of constant but different slopes is studied. Linear shallow-water theory is used for incoming impulse evolution, and nonlinear corrections are obtained for the run-up stage. It is demonstrated that bottom profile influences the run-up characteristics and can lead to resonance effects: increase of wave height, particle velocity, and number of oscillations. Simple parameterization of tsunami source through an earthquake magnitude is used to calculate the run-up height versus earthquake magnitude. It is shown that resonance effects lead to the sufficient increase of run-up heights for the weakest earthquakes, and a tsunami wave does not break on chosen bottom relief if the earthquake magnitude does not exceed 7.8.
\end{abstract}

\section{Introduction}

Resonance phenomena play a significant role in the run-up amplification and lead to different physical effects for waves in coastal zones: long duration of water oscillations, later arrival of waves with maximal amplitude compared with leading waves, and group structure of waves. Meanwhile, usually these effects are neglected when the run-up processes are studied. A large part of theoretical results for run-up stage are based on rigorous analytical solutions of the shallowwater theory for waves climbing on a beach of constant slope. This approach was suggested in the pioneer work by Carrier and Greenspan (1958). They applied the hodograph transformation to the nonlinear system of shallow-water equations and obtained a linear wave equation for an auxiliary function; all physical variables (free surface displacement, depthaveraged velocity, offshore coordinate and time) were explicitly expressed using this function and its partial derivatives. The main advantage of wave equations for an auxiliary function having a form of cylindrical wave equation is that it has to be solved on a semi-axis with given boundary conditions while the initial equations have to be solved in a domain with an unknown moving boundary (shoreline). Meanwhile, the explicit form of the analytical solution generally requires the numerical manipulations to present physical variables in the wave field. That is why various shapes of the incident solitary wave have been specially analyzed: soliton (Pedersen and Gjevik, 1983; Synolakis, 1987), sine pulse (Mazova et al., 1991), Lorentz pulse (Pelinovsky and Mazova, 1992), Gaussian pulse (Carrier et al., 2003; Kânoğlu, 2004; Kânoğlu and Synolakis, 2006), N waves (Tadepalli and Synolakis, 1994; Kânoğlu, 2004), and some specific localized disturbances (Tinti and Tonini, 2005; Pritchard and Dickinson, 2007; Dobrokhotov and Tirozzi, 2010). It should be noted that different formulas for maximum run-up of solitary waves of various shapes can be provided in terms of wave amplitude and significant wave length describing practically important cases with good accuracy (Didenkulova et al., 2008; Didenkulova and Pelinovsky, 2008; Antuono and Brocchini, 2010a, b). Various shapes of the periodic incident wave trains such as the sine wave (Carrier and Greenspan, 1958; Madsen and Fuhrman, 2008), cnoidal wave (Synolakis et al., 1988; Synolakis, 1991) and nonlinearly deformed periodic wave (Didenkulova et al., 2006, 2007) have been also 
studied to obtain the run-up characteristics. It is important to mention that the run-up height is higher if a periodic incident wave is cnoidal or a nonlinearly deformed wave compared with a simple sine wave of the same amplitude and period. Some results are obtained for irregular incident waves modeled by the Fourier superposition of the sine waves with random phases (Didenkulova et al., 2010, 2011) or the random set of solitons (Brocchini and Gentile, 2001).

In all studies mentioned above, the rigorous analytical solutions are obtained if the wave propagates on a plane beach of constant slope. Really, such a plane can approximate the face-shore bathymetry only, and then it has to be matched with a horizontal bottom profile. In fact, the rigorous analytical solutions can be obtained here in the linear theory only (Synolakis, 1987; Pelinovsky, 1996, 2006; Madsen and Fuhrman, 2008). If the bottom slope in face-shore area is small, the extreme run-up characteristics weakly differ from a case when the bottom has constant slope everywhere. Nonlinearity leads to the correction of obtained results. First of all, there is nonlinear wave deformation in the region where inclination of bottom changes. This effect is thoroughly investigated with use of boundary value approach for pulse-like and periodic input including waves propagating over different kinds of non-planar bathymetries in Brocchini et al. (2001) and Antuono and Brocchini (2007, 2008, $2010 \mathrm{~b}$ ). In this case the wave evolution seems to be unaffected by the bottom perturbations. The second one is that a wave moving on horizontal bottom nonlinearly deformed within nonlinear shallow-water equations as Riemann wave and its shape in the entry of plane beach differs from an initial shape (Didenkulova et al., 2006, 2007). It should be noted that both factors amplify the run-up heights. A new effect that appeared for the wave run-up on a plane beach matched with horizontal bottom is the influence of the bottom slope on the shape of water oscillations on the shore. If the incident wave has a bell shape, the water oscillations on the shore repeat its shape if the bottom slope is big (limiting case is a vertical wall), and accompanied by the negative second oscillation if the bottom slope is small. Such behavior is explained by the resonance effects, which are weak for such geometry - from a physical point of view, it is an open resonator ${ }^{1}$ (Pelinovsky, 1996, 2006; Madsen and Fuhrman, 2008). If the bottom slope differs relatively little from the uniform value, the changes of run-up height are also small (Soldini et al., 2013).

For more complicated geometry of coastal zone consisting of several pieces with different slopes, the solutions for each region of constant slope are matched (Kânoğlu and Synolakis, 1998; Didenkulova, 2009). Simplified solutions in the form of a product of such elementary solutions can be given if the incident wave length is less than a bottom piece length. For general ratio between these different lengths, as it is known, the resonances appear due to multi-reflection

\footnotetext{
${ }^{1}$ If the wave maker is located near the shore, of course, the resonant effects are big (Stefanakis et al., 2011; Ezersky et al., 2013).
}

from matching points and interference between such waves. Some allusion on possible resonances for wave run-up can be found in Kajiura (1977) and Mazova (1985). They investigated linear approximation of the run-up characteristics due to sine incident wave. Resonance phenomena are important for tsunami waves (LeBlond and Mysak, 1981; Massel, 1989; Mei, 1983; Neu and Shaw, 1987). Photos of tsunami wave trains in different coastal locations became well known after the 2004 Indonesian and 2011 Japanese tsunamis. Usually the appearance of resonance effects is connected with a complicated two-dimensional bathymetry of bays and jagged coastal line. In the present paper, we aim to investigate runup resonance phenomena for a one-dimensional case of wave propagation. We intend to show that, for certain frequencies depending on bottom profile, run-up amplification may be high even for very simple bathymetry, and it influences the shape of the water oscillations on the coast.

The paper is organized as follows. In the second section we describe our model of bottom profile and present the results for run-up amplification versus the frequency of linear harmonic incident wave. In the Sect. 3 results of calculations of the run-up characteristics caused by Gaussian impulse and $\mathrm{N}$-wave impulse are presented. Section 4 is devoted to nonlinear effects appearing in run-up. Discussion of result applicability for natural hazard description and some conclusions are given in Sect. 5.

\section{Theoretical model and run-up due to linear harmonic wave}

Long wave run-up on a long beach is described by 1-D nonlinear shallow water equations:

$$
\begin{aligned}
& \frac{\partial u}{\partial t}+u \frac{\partial u}{\partial x}+g \frac{\partial \eta}{\partial x}=0, \\
& \frac{\partial \eta}{\partial t}+\frac{\partial}{\partial x}[u(h+\eta)]=0,
\end{aligned}
$$

where $u$ is the depth-averaged velocity, $h=h(x)$ is the unperturbed water depth, $\eta=\eta(x, t)$ is the free surface displacement, and $g$ is the acceleration of gravity. In the linear approximation, the system in Eqs. (1)-(2) is transformed into one equation:

$\frac{\partial^{2} \eta}{\partial t^{2}}-g \frac{\partial}{\partial x}\left[h \frac{\partial \eta}{\partial x}\right]=0$.

To demonstrate the resonance effects in the run-up characteristics, we use three piece-wise profiles of unperturbed depths that are typical for a real ocean bottom: (Zone A) continental shelf $0 \leq x \leq x_{0}$, (Zone B) continental slope $x_{2}<x<0$, and (Zone C) constant depth (see Fig. 1). Such topography was used in numerous papers on tsunami run-up. We would like to emphasize that in some cases such a simple model of bottom profile describes very precisely natural conditions. For 


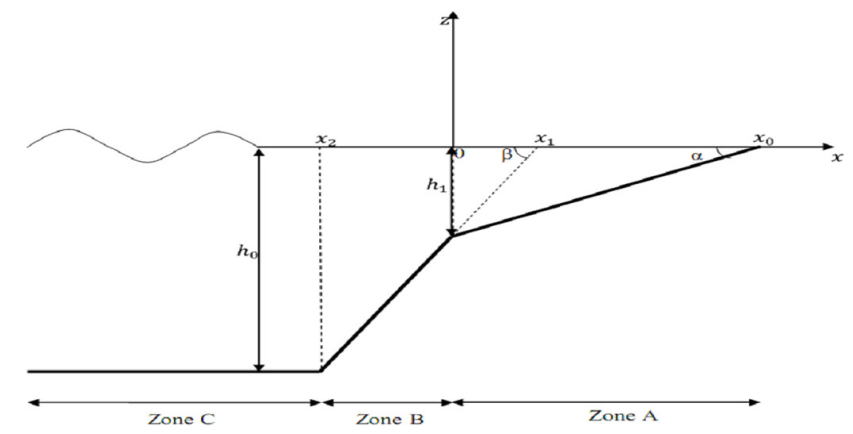

Fig. 1. Schema of bottom profile.

instance, exactly such a model was used to prepare numerical simulations of tsunami near the Indian coast (Neetu et al., 2011).

The wave field in zone of constant depth (Zone C) is presented as a sum of incident and reflected harmonic waves with constant amplitude $A_{\mathrm{i}}$ and $A_{\mathrm{r}}$ :

$\eta=\left(A_{\mathrm{i}} e^{-i k\left(x_{0}-x\right)}+A_{\mathrm{r}} e^{i k\left(x_{0}-x\right)}\right) e^{-i \omega t}$.

In zone (Zone B) with a constant bottom slope $\tan \beta$ (continental slope), we seek a harmonic solution of the following form:

$\eta=A(x) e^{-i \omega t}$,

where $\omega$ is the frequency and $A(x)$ is an amplitude function. By inserting Eq. (5) into Eq. (3), the amplitude equation for harmonic wave is represented as

$g \tan (\beta)\left(x_{1}-x\right) \frac{\partial^{2} A}{\partial x^{2}}-g \tan (\beta) \frac{\partial A}{\partial x}+\omega^{2} A=0$.

After introducing the variable transformation,

$\bar{\sigma}=2 \omega \sqrt{\frac{x_{1}-x}{g \tan \beta}}$.

Equation (6) can be simplified to the Bessel equation of the first kind:

$\bar{\sigma} \frac{\partial^{2} A}{\partial \bar{\sigma}^{2}}+\frac{\partial A}{\partial \bar{\sigma}}+\bar{\sigma} A=0$.

Its solution may be expressed as a sum of the zeroth order Bessel functions of the first $J_{0}$ and second $Y_{0}$ kinds with two constants $C_{1}$ and $C_{2}$ :

$A=C_{1} J_{0}(\bar{\sigma})+C_{2} Y_{0}(\bar{\sigma}), \bar{\sigma}=2 \omega \sqrt{\frac{x_{1}-x}{g \tan \beta}}$.

In the nearshore zone (Zone A), the solution for wave amplitude is also presented in Bessel functions. Taking into account that the wave field should be limited at the shore $\left(x=x_{0}\right)$, the wave amplitude is described by

$A=R J_{0}(\sigma), \quad \sigma=2 \omega \sqrt{\frac{x_{0}-x}{g \tan \alpha}}$,

where $R$ is also constant (in general, complex constant). It is evident that $R$ describes the amplitude of the water level oscillation on unmoved shoreline (linear run-up height). If the bottom has the constant slope everywhere, the value of $|R|$ computed in the linear theory coincides with run-up height in nonlinear theory (Synolakis, 1987; Pelinovsky and Mazova, 1992). For more complicated geometry, this statement is not proved and we will discuss this later.

Using continuity conditions for horizontal velocity and free surface displacement for $x=0$ and $x=x_{2}$ (see Fig. 1), one can match solutions in different segments at this transition points and obtain the following system of equations:

For $x=0$,

$R J_{0}\left(\sigma_{0}\right)=C_{1} J_{0}\left(\bar{\sigma}_{0}\right)+C_{2} Y_{0}\left(\bar{\sigma}_{0},\right)$
$R J_{1}\left(\sigma_{0}\right)=C_{1} J_{1}\left(\bar{\sigma}_{0}\right)+C_{2} Y_{1}\left(\bar{\sigma}_{0}.\right)$

For $x=x_{2}$,

$C_{1} J_{0}\left(\bar{\sigma}_{1}\right)+C_{2} Y_{0}\left(\bar{\sigma}_{1}\right)=A_{\mathrm{i}} e^{-i k\left(x_{0}-x_{2}\right)}+A_{\mathrm{r}} e^{i k\left(x_{0}-x_{2}\right)}$

$C_{1} J_{1}\left(\bar{\sigma}_{1}\right)+C_{2} Y_{1}\left(\bar{\sigma}_{1}\right)=-i A_{\mathrm{i}} e^{-i k\left(x_{0}-x_{2}\right)}-i A_{\mathrm{r}} e^{i k\left(x_{0}-x_{2}\right)}$

where $\sigma_{0}=\sigma(x=0), \bar{\sigma}_{0}=\bar{\sigma}(x=0), \bar{\sigma}_{1}=\bar{\sigma}\left(x=x_{2}\right)$, and $J_{1}$ and $Y_{1}$ are the first-order Bessel functions of first and second kinds.

If the incident wave amplitude $A_{\mathrm{i}}$ is known, the linear runup height $R$ can be evaluated by solving the last system of Eqs. (11)-(14):

$R=K(\omega) A_{\mathrm{i}}$

$K(\omega)=\frac{2\left(J_{0}\left(\bar{\sigma}_{0}\right) Y_{1}\left(\bar{\sigma}_{0}\right)-J_{1}\left(\bar{\sigma}_{0}\right) Y_{0}\left(\bar{\sigma}_{0}\right)\right)}{W\left(Y_{0}\left(\bar{\sigma}_{1}\right)-i Y_{1}\left(\bar{\sigma}_{1}\right)\right)-N\left(J_{0}\left(\bar{\sigma}_{1}\right)-i J_{1}\left(\bar{\sigma}_{1}\right)\right)} e^{-i k\left(x_{0}-x_{2}\right)}$

where

$W=J_{0}\left(\bar{\sigma}_{0}\right) J_{1}\left(\sigma_{0}\right)-J_{0}\left(\sigma_{0}\right) J_{1}\left(\bar{\sigma}_{0}\right)$,

$N=Y_{0}\left(\bar{\sigma}_{0}\right) J_{1}\left(\sigma_{0}\right)-J_{0}\left(\sigma_{0}\right) Y_{1}\left(\bar{\sigma}_{0}\right)$.

The term in numerator of Eq. (15) may be simplified using Wronskian (Abramovich and Stegun, 1964) as presented in Kânoglu and Synolakis (1998):

$J_{0}\left(\bar{\sigma}_{0}\right) Y_{1}\left(\bar{\sigma}_{0}\right)-J_{1}\left(\bar{\sigma}_{0}\right) Y_{0}\left(\bar{\sigma}_{0}\right)=-\frac{2}{\pi \bar{\sigma}_{0}}$.

Figure 2 represents run-up amplification $|R| / A_{\mathrm{i}}$ for three different sets of bottom slopes characterized roughly for the Indian coast bathymetry (Neetu et al., 2011) where the Makran tsunami was observed on 27 November 1945. If the bottom slopes in zones A and B are the same, the resonance effects are very weak (dash-point curve in Fig. 2), and this coincides 


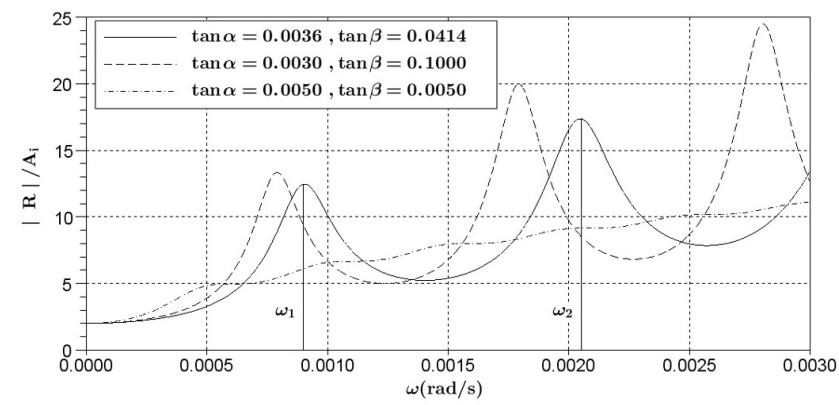

Fig. 2. Run-up amplifications for three sets of bottom slopes, $h_{0}=$ $2500 \mathrm{~m}, h_{1}=200 \mathrm{~m}$.

with known results (Pelinovsky, 1996, 2006; Madsen and Fuhrman, 2008). But in the case of different bottom slopes in zones A and B, resonance effects are clearly visible (solid and dashed lines in Fig. 2). Several resonant modes with frequencies $\left(\omega_{1}, \omega_{2}, \ldots\right)$ may be excited in the coastal zone, and the amplification coefficient can reach values of 10-20 times.

Characteristic period of the first resonant peak $T=2 \pi / \omega_{1}$ is roughly $2 \mathrm{~h}$, which coincides with the observed tsunami record in this area (1.5-3 h) according to Neetu et al. (2011).

\section{Run-up due of solitary bell and $N$ impulses}

Resonance curves given in the previous section show a substantial increase of run-up heights for certain frequencies of harmonic incident waves. Whereas run-up height for harmonic waves is given by Eq. (15), the oscillations of water level on the shore (linear run-up) generated by solitary tsunami wave may be presented using Fourier transformations:

$R(t)=\frac{1}{2 \pi} \int K(\omega) S(\omega) e^{(-i \omega t)} \mathrm{d} \omega$,

where $S$ is the Fourier transformation of incident wave

$S(\omega)=\int \eta(t) e^{(i \omega t)} \mathrm{d} t$.

Usually the shape of the incident tsunami wave is unknown, and it is characterized by different functions (see for instance Didenkulova et al., 2008). We chose here two characteristic and qualitatively different cases: the initial displacement of the free surface of one sign and alternating displacement with a zero averaged value. In the first case the incoming wave at the point $x=x_{2}$ is a Gaussian pulse, and in the second one a so-called $N$ pulse:

$\eta_{\text {in } G}=\eta_{0} e^{\left(-\left(t / \tau_{0}\right)^{2}\right)}$,

$\eta_{\text {in } N}=\eta_{0}\left(\frac{t}{\tau_{0}}\right) e^{\left(-\left(t / \tau_{0}\right)^{2}\right)}$.
Both pulses are characterized by two parameters: the duration and amplitude. Within linear theory, the value of wave amplitude is not important and can be used for scaling of runup characteristics. The second parameter, wave duration $\tau_{0}$, plays an important role due to resonance effects.

Meanwhile, in tsunami practice, both parameters (amplitude and duration) of the incident wave are not independent and are determined by the parameters of the tsunami source. Here we apply our theoretical results to tsunamis generated by underwater earthquakes. Now, the characteristics of the tsunami source are calculated using the Okada solution (Okada, 1985), and they depend on the several fault parameters. For simplified estimates it is more convenient to have the relations between tsunami source parameters and earthquake magnitude. Such relations are known in seismology (Sato, 1979; Wells and Coppersmith, 1994). Similar relations are given for parameters of tsunami source (Pelinovsky, 1996, 2006; Bolshakova and Nosov, 2011). Here we will use the following relations between the displacement amplitude of the free water surface $\eta_{0}$ (measured in meters) and the characteristic size of tsunami source $L$ (measured in $\mathrm{km}$ ) with the earthquake magnitude $M$ (Pelinovsky, 1996, 2006):

$\begin{aligned} \log \left(\eta_{0}\right) & =0.8 M-5.6 \\ \log (L) & =0.5 M-2.2\end{aligned}$

In the shallow-water approximation, the duration of tsunami waves going out the source is $\tau_{0}=L / \sqrt{g h}$, where $h$ is water depth in the tsunami source. Of course, Eqs. (20) and (21) are very approximated and should be used only for simplified estimations.

Thus, we can use the magnitude of a earthquake to describe solitary bell, or $\mathrm{N}$ wave. The results of calculations of linear run-up function $R(t)$ for various values of the earthquake magnitude are presented in Fig. 3. Duration of the incident tsunami waves decreases, and then magnitude decreases, and its spectrum width increases. This means that the weakest earthquake induces more resonant modes in coastal zones than the strongest earthquake. As a result, the number of water oscillations on the shore increases with magnitude decreasing (compare Fig. 3a and b). If the initial shape is $\mathrm{N}$ wave, the number of oscillations is higher than for Gaussian input because its spectrum is narrower (compare right and left graphs in Fig. 3a). It is important to mention that run-up height of $\mathrm{N}$ waves is higher than for bell waves, and this was obtained firstly in Tadepalli and Synolakis (1994). Using a simple formula, it is possible to estimate propagation time $T_{\Delta}$ (delay between incoming wave and computed signal at the coast) for our model.

$T_{\Delta}=\frac{2}{\tan \beta}\left(\sqrt{\frac{h_{0}}{g}}-\sqrt{\frac{h_{1}}{g}}\right)+\frac{2}{\tan \alpha} \sqrt{\frac{h_{1}}{g}}$.

The propagation time for parameters corresponding to Fig. 3 is estimated as $T_{\Delta} \approx 10^{3} \mathrm{~s}$. It should be noted that for longer 

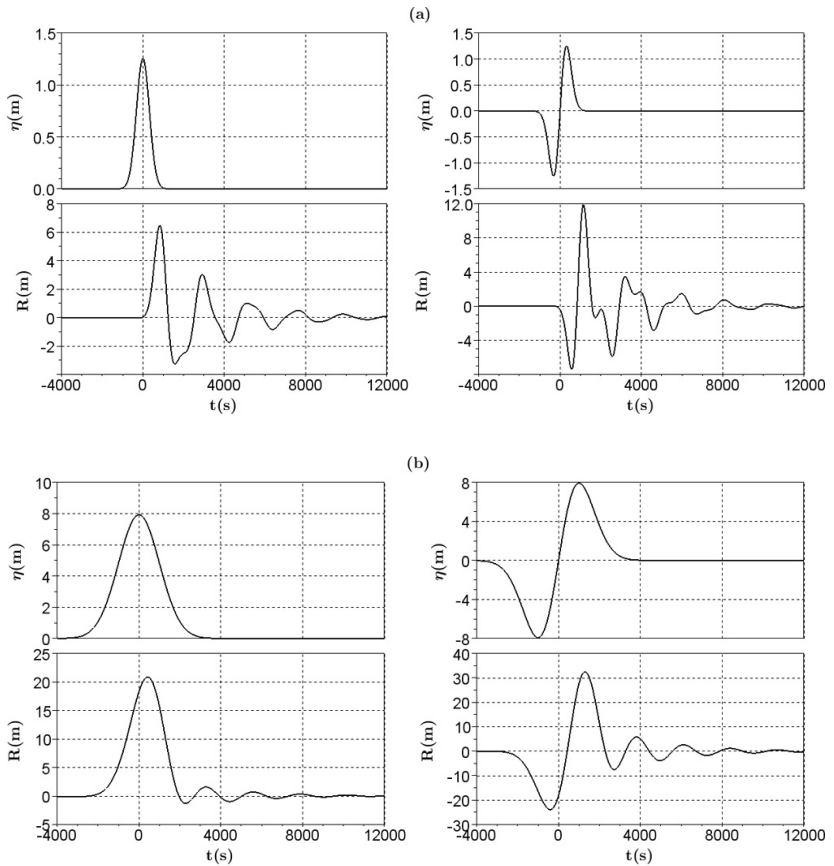

Fig. 3. Incident wave and water oscillations on the shore for $h_{0}=2500 \mathrm{~m}, x_{2}=23 \mathrm{~km}, h_{1}=150 \mathrm{~m}, x_{0}=15 \mathrm{~km}, \tan \alpha=0.005$, $\tan \beta=0.1$, and different earthquake magnitudes: (a) $M=7.5$ $(L=35 \mathrm{~km})$ and (b) $M=8.5(L=110 \mathrm{~km})$.

impulses (Fig. 3b), calculated propagation time $\left(T_{\Delta} \approx 450 \mathrm{~s}\right)$ is less than for shorter impulses $\left(T_{\Delta} \approx 850 \mathrm{~s}\right)$ (Fig. 3a). This difference is evidently due to duration of impulses. The length of a long impulse is longer than the length of a shelf zone, and reflected waves should be taken into consideration when propagation time is calculated as a time needed for impulse maximum, or impulse zero to reach the shore.

Figure 4 demonstrates that the run-up amplification factor $\left(R / \eta_{0}\right)$ decreases with increasing earthquake magnitude up to $M=7$, and then it remains almost constant. A tsunami generated by strong earthquake has a long wavelength, and in this case as indicated in Fig. 4, the resonance effects are very weak. The weakest tsunamis having the shortest wavelength are amplified more due to resonance effects. Increasing of the bottom slope in zone $\mathrm{B}(\tan \beta)$ reduces the run-up height as it might be expected.

Maximal run-up height grows with earthquake magnitude increasing as it is shown in Fig. 5. It should be noted that resonance effects "lift up" the values of run-up height for the weakest earthquake, whereas run-up height weakly depends on the values of the bottom slopes in the given ranges under consideration. It is important to note that curves in Figs. 4 and 5 are obtained in the linear approximation. Criteria for applicability of the linear solution will be discussed in the next section.
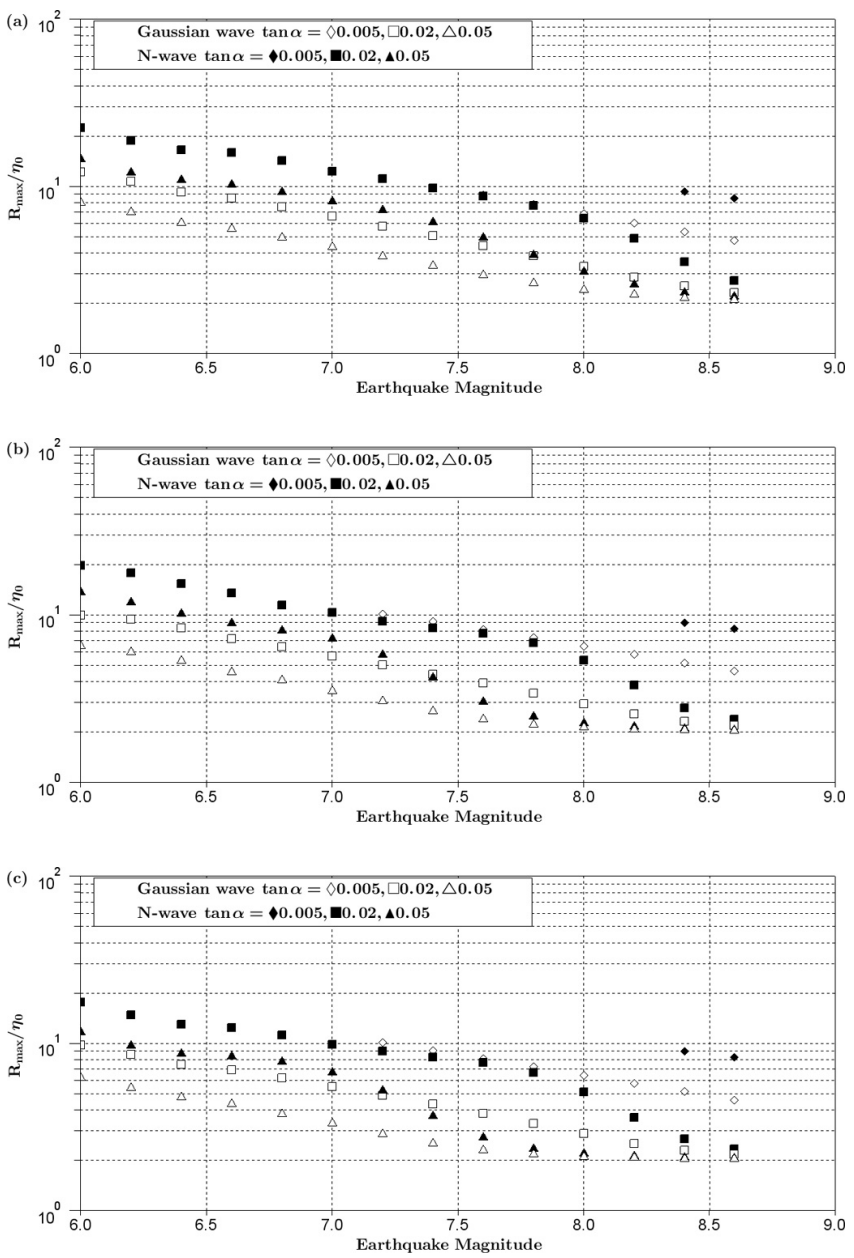

Fig. 4. Run-up amplification factor for Gaussian impulses and $\mathrm{N}$ wave impulse $x_{0}=40 \mathrm{~km}(\tan \alpha=0.005), x_{0}=10 \mathrm{~km}(\tan \alpha=$ $0.02), x_{0}=4 \mathrm{~km}(\tan \alpha=0.05), h_{0}=4000 \mathrm{~m}$, and $h_{1}=200 \mathrm{~m}$ for different inclinations of continental shelf and continental slope: (a) $\tan \beta=0.1 \quad\left(x_{2}=40 \mathrm{~km}\right)$, (b) $\tan \beta=0.5\left(x_{2}=8 \mathrm{~km}\right)$, and (c) $\tan \beta=3\left(x_{2}=1.3 \mathrm{~km}\right)$.

\section{Estimations of nonlinear effects}

Calculations of maximal run-up heights in Sects. 2 and 3 were done in linear approximation. As it is indicated above, it is difficult to solve the nonlinear shallow-water equations for piece-wise bottom profiles. Taking into account that bottom slope is changed at depth of 4000 and $200 \mathrm{~m}$, and wave amplitude does not exceed a few meters, we may assume that all nonlinear effects are manifested in the last run-up stage. In this case we may use the rigorous solution of the nonlinear shallow-water equations for the long wave run-up on a beach of constant slope, which is very well developed (see references in Introduction). Here following Pelinovsky and Mazova (1992), we convert the obtained linear solution into a "nonlinear" solution. According to this procedure, we should firstly find a "linear" expression for horizontal velocity on the 
unmoved shoreline $(x=0)$, which is followed by kinematics:

$U(t)=\frac{1}{\tan \alpha} \frac{\mathrm{d} R}{\mathrm{~d} t}$,

where as earlier $\tan \alpha$ is bottom face-slope. "Nonlinear" velocity of the moving shoreline, $u(t)$, can be obtained from the linear function $U(t)$ by the Riemann transformation (Pelinovsky and Mazova, 1992):

$u(t)=U\left(t+\frac{u}{\operatorname{gtan} \alpha}\right)$.

It is evident that maximal values of "nonlinear" and "linear" velocities coincide.

Vertical displacement of the moving shoreline, $r(t)$ can be found from a kinematic condition:

$r(t)=\frac{\int u(t) \mathrm{d} t}{\tan \alpha}$.

And after, substitution of Eq. (16) can be reduced to

$r(t)=R\left(t+\frac{u}{\tan \alpha g}\right)-\frac{u^{2}(t)}{2 g}$.

It should be noted that this is only true for the analytical structure of the solution, but the solution itself also depends on the data assignment as an initial value or a boundary value problem (Antuono and Brocchini, 2007).

The important conclusion from Eq. (24) is that extremes of the vertical displacement in the linear and nonlinear theories coincide (in this moment the horizontal velocity $u=0$ ), confirming the use of linear theory to predict extreme values. Therefore, the linear theory adequately describes the run-up height.

Simple formulas of Riemann transformation from linear to nonlinear solutions allow us to obtain the wave-breaking criterion. Strictly speaking, this criterion is found from the zero condition for a Jacobian of hodograph (Legendre) transformation. Note that this transformation was used to obtain Eqs. (15)-(17). On the other hand, the solution for velocity in Eq. (16) resembles the well-know Riemann wave in nonlinear acoustics and hydrodynamics (the role of coordinates plays the inverse value of the $g \tan \alpha$ ). Such a wave would overturn with an increase of amplitude. Exactly this fact has been used in Pelinovsky $(1996,2006)$ and Didenkulova (2009) to find wave-breaking criteria on the shore. From Eq. (16) it is easy to calculate the time derivative of the velocity in an incident wave.

$\frac{\mathrm{d} u}{\mathrm{~d} t}=\frac{\mathrm{d} U / \mathrm{d} t}{1-\frac{\mathrm{d} U / \mathrm{d} t}{g \tan \alpha}}$

tends to the infinity when the denominator approaches zero. As follows from the theory of hyperbolic equations, it leads to the gradient catastrophe identified and to the plunging
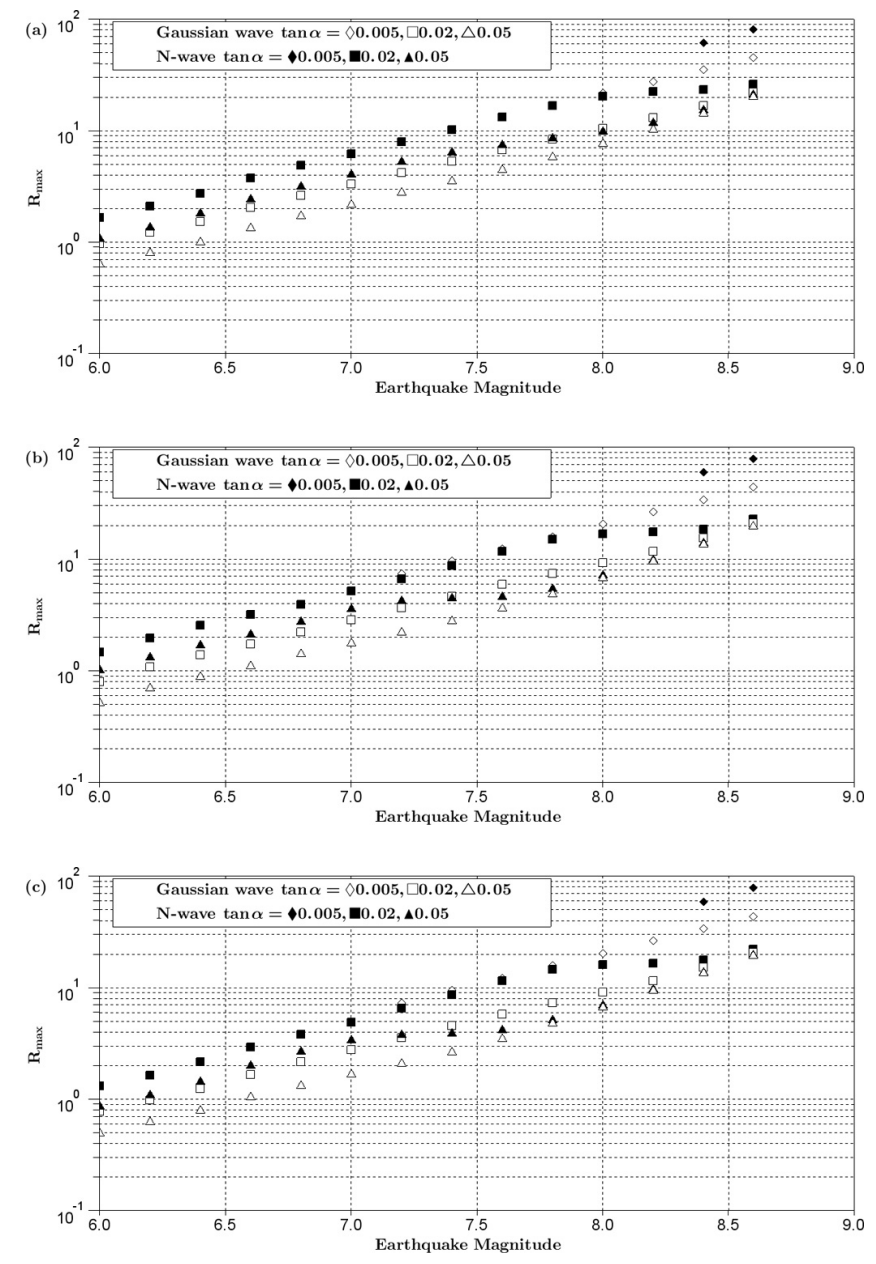

Fig. 5. Maximal run-up height (in $\mathrm{m}$ ) versus the earthquake magnitude for Gaussian impulses and $\mathrm{N}$ wave impulse for $x 0=40 \mathrm{~km}$ $(\tan \alpha=0.005), x_{0}=10 \mathrm{~km}(\tan \alpha=0.02), x_{0}=4 \mathrm{~km} \quad(\tan \alpha=$ $0.05), h_{0}=4000 \mathrm{~m}$, and $h_{1}=200 \mathrm{~m}$ for different inclinations of continental shelf and continental slope: (a) $\tan \alpha=0.1 \quad\left(x_{2}=\right.$ $40 \mathrm{~km})$, (b) $\tan \alpha=0.5\left(x_{2}=8 \mathrm{~km}\right)$, and (c) $\tan \alpha=3 \quad\left(x_{2}=\right.$ $1.3 \mathrm{~km})$.

breaking of the long water waves. In this case a water displacement contains the jump of its first derivative. This implies the condition of the first wave breaking:

$\mathrm{Br}=\frac{\max (\mathrm{d} U / \mathrm{d} t)}{\tan \alpha g}=\frac{\max \left(\mathrm{d}^{2} R / \mathrm{d} t^{2}\right)}{\tan ^{2} \alpha g}=1$,

where the parameter $\mathrm{Br}$ has the sense of breaking parameters. Figure 6 shows the temporal evolution of the breaking parameter, linear and nonlinear water level oscillations on the shore and shoreline velocities versus time for solitary impulse and $N$ wave impulse for magnitude $M=8$. The difference is clearly seen between linear and nonlinear solutions for a moving shoreline. It is important to mention that the breaking parameter is less than 1 , so the tsunami wave should climb on the shore without breaking for the chosen 

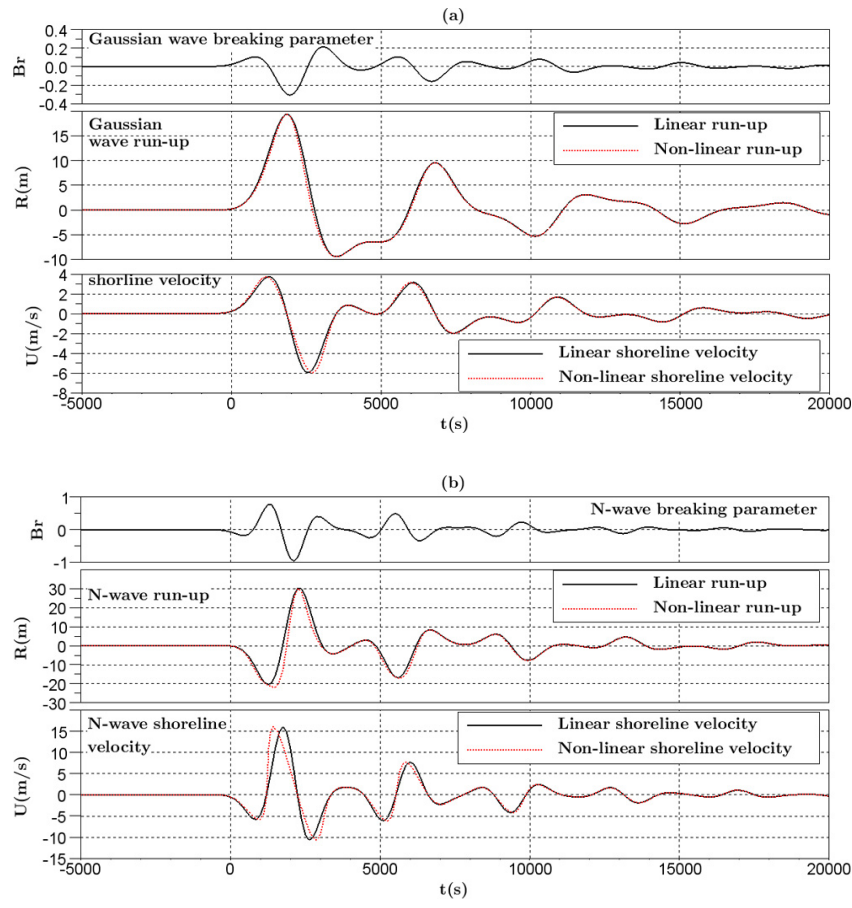

Fig. 6. Breaking parameter $\mathrm{Br}$, linear (solid line) and nonlinear (dash line) variations of water level $\mathrm{R}$ on shore and shoreline velocities $U$ versus time for $\mathrm{h} 0=4000 \mathrm{~m}, \mathrm{~h} 1=200 \mathrm{~m}, \tan \alpha=0.005, x_{0}=$ $40 \mathrm{~km}, \tan \alpha=0.1, x_{2}=23 \mathrm{~km}$, and $M=8$ : (a) Gaussian wave, and (b) $\mathrm{N}$ wave.

bottom geometry. It should be emphasized that for all results presented in Figs. 4 and 5 criterion $\mathrm{Br}<1$ is satisfied.

\section{Discussion and conclusions}

The run-up of tsunami waves on the coast is studied for the following bottom geometry: ocean of constant depth, steep continental slope, and beach of gentle constant slope. It is demonstrated that run-up characteristics strongly depend on the frequency of the incident wave due to resonance effects. They are studied for conditions of the Indian coast where the 1945 Makran tsunami was recorded. Amplification ratio can be 10 times higher than for a case of a uniform, averaged slope. The run-ups of solitary waves of bell or $\mathrm{N}$ shape are studied in detail. It is found that run-up of $\mathrm{N}$ wave is higher than for solitary waves. This is due to stronger manifestation of the resonance effects for $\mathrm{N}$ wave than for bell-shaped waves. Using simple parameterization of tsunami source through an earthquake magnitude, the runup heights are calculated versus earthquake magnitude. It is shown that the resonance effects can "lift up" the values of run-up heights for the weakest magnitudes due to resonance amplification of the shortest waves generated by the weakest earthquake. Nonlinear correction of obtained results is given. It is shown that, for typical conditions of the Indian coast where the 1945 Makran tsunami was observed, the breaking parameter is less than 1 , and tsunami waves climb on the coast with no breaking.

Acknowledgements. Efim Pelinovsky thanks partial supports from RFBR grant (11-05-00216), Austrian Science Foundation (FWF) under project P 246710, Federal Target Program "Scientific-Pedagogical Personnel of Innovative Russia for 20092013", VolkswagenStiftung, and highly appreciates the offered opportunity to work as a visiting professor at M2C 6143 CNRS laboratory of Caen University. Alexander Ezersky thanks Anjan Kundu for invitation and fruitful discussions on tsunami resonance effects during visit the Saha Institute of Nuclear Physics in Calcutta.

Edited by: S. Tinti

Reviewed by: M. Brocchini and two anonymous referees

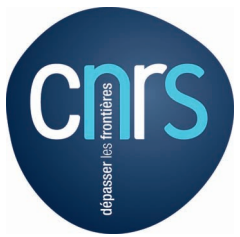

The publication of this article is financed by CNRS-INSU.

\section{References}

Abramowitz, M. and Stegun, I. A.: Handbook of Mathematical Functions with Formulas, Graphs and Mathematical Tables, Vol. 55, National Bureau of Standards Applied Mathematics Series, US Government Printing Office, Washington, DC, 1964.

Antuono, M. and Brocchini, M.: The boundary value problem for the nonlinear shallow water equations, Stud. Appl. Math., 119, 73-93, doi:10.1111/j.1365-2966.2007.00378.x, 2007.

Antuono, M. and Brocchini, M.: Maximum run-up, breaking conditions and dynamical forces in the swash zone: a boundary value approach, Coast. Eng., 55, 732-740, doi:10.1016/j.coastaleng.2008.02.002, 2008.

Antuono, M. and Brocchini, M.: Analysis of the Nonlinear Shallow Water Equations Over Nonplanar Topography, Stud. Appl. Math., 124, 85-103, doi:10.1111/j.1467-9590.2009.00464.x, 2010a.

Antuono, M. and Brocchini, M.: Solving the nonlinear shallowwater equations in physical space, J. Fluid Mech., 643, 207-232, doi:10.1017/S0022112009992096, 2010b.

Bolshakova, A. and Nosov, M.: Parameters of tsunami source versus earthquake magnitude, Pure Appl. Geophys., 168, 2023-2031, doi:10.1007/s00024-011-0285-3, 2011.

Brocchini, M. and Gentile, R.: Modelling the run-up of significant wave groups, Cont. Shelf Res., 21, 1533-1550, doi:10.1016/S0278-4343(01)00015-2, 2001.

Brocchini, M., Bernetti, R., Mancinelli, A., and Albertini, G.: An efficient solver for nearshore flows based on the WAF method, Coast. Eng., 43, 105-129, 2001.

Carrier, G. F. and Greenspan, H. P.: Water waves of finite amplitude on a sloping beach, J. Fluid Mech., 4, 97-109, doi:10.1017/S0022112058000331, 1958. 
Carrier, G. F., Wu, T. T., and Yeh, H.: Tsunami run-up and draw-down on a plane beach, J. Fluid Mech., 475, 79-99, doi:10.1017/S0022112002002653, 2003.

Didenkulova, I.: Nonlinear long-wave deformation and runup in a basin of varying depth, Nonlin. Processes Geophys., 16, 23-32, doi:10.5194/npg-16-23-2009, 2009.

Didenkulova, I. and Pelinovsky, E. N.: Run-up of long waves on a beach: The influence of the incident wave form, Oceanology, 48, 1-6, doi:10.1134/S0001437008010013, 2008.

Didenkulova, I., Pelinovsky, E., Soomere, T., and Zahibo, N.: Runup of nonlinear asymmetric waves on a plane beach, in: Tsunami and Nonlinear Waves, edited by: Kundu, A., Springer Berlin Heidelberg, 175-190, doi:10.1007/978-3-540-71256-5_8, 2007.

Didenkulova, I., Pelinovsky, E., and Soomere, T.: Runup characteristics of symmetrical solitary tsunami waves of "unknown" shapes, Pure Appl. Geophys., 165, 2249-2264, doi:10.1007/s00024-008-0425-6, 2008.

Didenkulova, I., Pelinovsky, E., and Sergeeva, A.: Statistical characteristics of long waves nearshore, Coast. Eng., 58, 94-102, doi:10.1016/j.coastaleng.2010.08.005, 2011.

Didenkulova, I. I., Zahibo, N., Kurkin, A. A., Levin, B. V., Pelinovsky, E. N., and Soomere, T.: Runup of nonlinearly deformed waves on a coast, Dokl. Earth Sci., 411, 1241-1243, doi:10.1134/S1028334X06080186, 2006.

Didenkulova, I. I., Sergeeva, A. V., Pelinovsky, E. N., and Gurbatov, S. N.: Statistical estimates of characteristics of long-wave run-up on a beach, Izv. Atmos. Oceanic Phy+, 46, 530-532, doi:10.1134/S0001433810040122, 2010.

Dobrokhotov, S. Y. and Tirozzi, B.: Localized solutions of one-dimensional non-linear shallow-water equations with velocity $c=(x)^{1 / 2}$, Russ. Math. Surv., 65, 177-179, doi:10.1070/RM2010v065n01ABEH004668, 2010.

Ezersky, A., Abcha, N., and Pelinovsky, E.: Physical simulation of resonant wave run-up on a beach, Nonlin. Processes Geophys., 20, 35-40, doi:10.5194/npg-20-35-2013, 2013.

Kajiura, K.: Local behaviour of tsunamis, in: Waves on Water of Variable Depth, edited by: Provis, D. G. and Radok, R., Springer Berlin Heidelberg, Lect. Notes Phys, 64, 72-79, doi:10.1007/3540082530_137, 1977.

Kânoğlu, U.: Nonlinear evolution and runup and rundown of long waves over a sloping beach, J. Fluid Mechanics, 513, 363-72, doi:10.1017/S002211200400970X, 2004.

Kânoğlu, U. and Synolakis, C.: Long wave run-up on piecewise liner topographies, J. Fluid Mech., 374, 1-28, 1998.

Kânoğlu, U. and Synolakis, C.: Initial value problem solution of nonlinear shallow water-wave equations, Phys. Rev. Lett., 97, 148501, doi:10.1103/PhysRevLett.97.148501, 2006.

LeBlond, P. H. and Mysak, L. A.: Waves in the Ocean, Vol. 20, Elsevier Science, Amsterdam, 1981.

Madsen, P. A. and Fuhrman, D. R.: Run-up of tsunamis and long waves in terms of surf-similarity, Coast. Eng., 55, 209-223, doi:10.1016/j.coastaleng.2007.09.007, 2008.

Massel, S. R.: Hydrodynamics of Coastal Zones, Vol. 48, Elsevier Science, 1989.

Mazova, R. K.: Linear theory of the wave runup on shelves of different geometries, in: Wave Runup on Beach, edited by: Pelinovsky, E., Institute of Applied Physics, Gorky, 48-63, 1985.
Mazova, R. K., Osipenko, N. N., and Pelinovsky, E. N.: Solitary wave climbing a beach without breaking, Rozprawy Hydrotechniczne, 54, 71-80, 1991.

Mei, C. C.: The Applied Dynamics of Ocean Surface Waves, Wiley, New York, USA, 1983.

Neetu, S., Suresh, I., Shankar, R., Nagarajan, B., Sharma, R., Shenoi, S. S. C., Unnikrishnan, A. S., and Sundar, D.: Trapped waves of the 27 November 1945 Makran tsunami: observations and numerical modeling, Nat. Hazards, 59, 1609-1618, doi:10.1007/s11069-011-9854-0, 2011.

Neu, W. L. and Shaw, R. P.: Tsunami filtering by ocean topography, Ocean Physics and Engineering, 12, 1-23, 1987.

Okada, Y.: Surface deformation due to shear and tensile faults in a half-space, B. Seismol. Soc. Am., 75, 1135-1154, 1985.

Pedersen, G. and Gjevik, B.: Run-up of solitary waves, J. Fluid Mechanics, 135, 283-299, doi:10.1017/S0022112083003080, 1983.

Pelinovsky, E.: Hydrodynamics of tsunami waves, in: Waves in geophysical fluids, edited by: Grue, J. and Trulsen, K., Tsunamis, Rogue Waves, Internal Waves and Internal Tides, CISM International Centre for Mechanical Sciences, Springer, 489, 1-48, 2006.

Pelinovsky, E. N.: Hydrodynamics of Tsunami Waves, Applied Physics Institute Press, Nizhny Novgorod, 1996.

Pelinovsky, E. N. and Mazova, R. K.: Exact analytical solutions of nonlinear problems of tsunami wave run-up on slopes with different profiles, Nat. Hazards, 6, 227-249, doi:10.1007/BF00129510, 1992.

Pritchard, D. and Dickinson, L.: The near-shore behaviour of shallow-water waves with localized initial conditions, J. Fluid Mech., 591, 413-436, doi:10.1017/S002211200700835X, 2007.

Sato, R.: Theoretical basis on relationships between focal parameters and earthquake magnitude, J. Phys. Earth, 27, 353-372, 1979.

Soldini, L., Antuono, M., and Brocchini, M.: Numerical modeling of the influence of the beach profile on wave run-up, J. Waterw. Port C.-ASCE, 139, 61-71, doi:10.1061/(ASCE)WW.19435460.0000163, 2013.

Stefanakis, T. S., Dias, F., and Dutykh, D.: Local run-up amplification by resonant wave interactions, Phys. Rev. Lett., 107, 124502, doi:10.1103/PhysRevLett.107.124502, 2011.

Synolakis, C. E.: The runup of solitary waves, J. Fluid Mech., 185, 523-545, doi:10.1017/S002211208700329X, 1987.

Synolakis, C. E.: Tsunami runup on steep slopes: How good linear theory really is, Nat. Hazards, 4, 221-234, doi:10.1007/BF00162789, 1991.

Synolakis, C. E., Deb, M. K., and Skjelbreia, J. E.: The anomalous behavior of the runup of cnoidal waves, Phys. Fluids, 31, 3-5, doi:10.1063/1.866575, 1988.

Tadepalli, S. and Synolakis, C. E.: The run-up of N-waves on sloping beaches, Proc. R. Soc. Lon. Ser.-A, 445, 99-112, doi:10.1098/rspa.1994.0050, 1994.

Tinti, S. and Tonini, R.: Analytical evolution of tsunamis induced by near-shore earthquakes on a constant-slope ocean, J. Fluid Mechanics, 535, 33-64, doi:10.1017/S0022112005004532, 2005.

Wells, D. L. and Coppersmith, K. J.: New empirical relationships among magnitude, rupture length, rupture width, rupture area, and surface displacement, B. Seismol. Soc. Am., 84, 974-1002, 1994. 